\title{
Session VI
}

\section{Evolutionary Aspects}

chair: C. Sterken 


\title{
The Evolution of Non-spherical and Non-stationary Winds of Massive Stars
}

\author{
Norbert Langer \\ Institut für Physik, Universität Potsdam, D-14415 Potsdam, Germany
}

\begin{abstract}
We describe present theoretical ideas about the time evolution of the winds of luminous stars with emphasis to effects of non-sphericity and non-stationarity. We discuss the evolution of the winds of rotating luminous stars during their main sequence evolution, in particular when they approach their Eddington-limit or any other surface instability. We then consider the winds of post-main sequence stars up to the immediate pre-supernova stage. We connect the giant outbursts of Luminous Blue Variables with luminous rotating post-main sequence stars in thermal disequilibrium. We further discuss the spin-up effect of Heger \& Langer (1998) for post-red supergiants and describe its observational consequences. We compare theoretical models with observations of the winds of $\mathrm{B}[\mathrm{e}]$ supergiants and Luminous Blue Variables in general, and with SN 1987A, VY CMa and $\eta$ Car and the Pistol Star in particular.
\end{abstract}

\section{Introduction}

Massive stars $\left(M \gtrsim 10 \mathrm{M}_{\odot}\right)$ are very luminous $\left(L \gtrsim 10^{4} \mathrm{~L}_{\odot}\right)$ and have strong winds. During their evolution, their surface properties change which gives rise to changes in their wind properties. For example, the terminal wind speed is always of the order of the escape velocity at the stellar surface (Cassinelli \& Lamers 1998). Therefore, as the massive stars move across the HR diagram during their post-main sequence evolution, the speed of their winds are changing dramatically. For contracting stars, i.e., for an increasing wind speed, this leads to swept-up dense shells around stars which then can form bright visible nebulae -- this concerns in particular LBVs and post-red supergiants; cf. García-Segura et al. (1996ab) - much like in the case of planetary nebulae around low-mass post-AGB stars.

As important as the time dependence of the stellar wind properties is their spatial structure. In fact, there is compelling evidence that the winds of massive stars are not generally isotropic (cf. below).

In this paper, we focus on non-sphericity and non-stationarity of massive star winds, but referring always to large spatial and time scales. I.e., the shortest variability time scales which we consider here is that of the thermal time scale of the stellar envelope - which can be as short as one year - while we generally do not consider variability due to stellar pulsations (although see Section 5) or due to rotation. And while we explicitly consider the effect of rotation on the spatial structure of the wind, we do not discuss small scale features as clumping or magnetic star spots. 


\section{Assumptions}

As theoretical models for the envelopes and atmospheres of rapidly rotating luminous stars do not yet exist, we have to make several assumptions in the following. Let us consider a rotating star which, either due to increasing luminosity or - more realistically - due to increasing surface opacity, approaches its Eddington limit. Whether or not the stellar luminosity and the equatorial radiation flux are strongly affected by the rotation is unclear at the moment. Even though it is sometimes assumed that the von Zeipel (1924) theorem on rotational or "gravity darkening" can be applied (e.g., Owocki et al. 1998, Glatzel 1998), it may give spurious results for stars considered here, in which only the outer "skin" ( $\sim 0.01 \%$ of the stellar mass) is actually close to critical rotation, the energy transport in these layers is largely due to convection, and the internal circulation pattern is unknown. In fact, Kippenhahn (1977) has shown that even solutions for which the radiative flux increases with decreasing gravity ("gravity brightening") can be obtained. Thus, even though it can not be excluded that rapidly rotating stars blow a denser wind along their polar axis than in their equatorial plane as a result of "gravity darkening" (Owocki et al. 1996), we rather assume a constant surface brightness of the star, and consequently an outflow preferentially in the equatorial plane for the rapidly rotating stars considered here. Were the star non-rotating, its surface would become unstable when the Eddington factor $\Gamma=L / L_{\text {edd }}=\kappa L /(4 \pi c G M)$ approaches the critical value of one. However, when the star is rotating, the centrifugal force has to be considered in the force balance. Assuming an unchanged radiation flux as function of latitude, instability occurs at the stellar equator actually for $\Omega \rightarrow 1$ (with $\Omega=v_{\text {rot }} / v_{\text {crit }}$ and $v_{\text {crit }}^{2}=G M(1-\Gamma) / R ;$ cf. Langer 1997) at a value of $\Gamma<1$ (cf. Fig. 1).

We want to emphasize that the principle of the so called $\Omega$-limit (instead of Eddington-limit) is more general: Any surface instability in a non-rotating star - e.g. the turbulent pressure instability investigated by Nieuwenhuijzen and de Jager (1995) - will be affected by rotation such that the spherical symmetry is broken and mass outflow will be strongly latitude-dependent -. i.e., critical rotation is reached (cf. Langer 1998).

A further assumption made in the following concerns the time dependence of the obtained mass loss. As mentioned in Sect. 1, we will ignore here variability on short time scales. Furthermore, we will assume that for a star which approaches the surface instability the mass loss increases drastically (Friend \& Abbott 1986, Owocki et al. 1996), but remains limited to values of the order of $\dot{M} \sim M_{\mathrm{env}} / \tau_{\mathrm{evol}}$, where $M_{\mathrm{env}}$ is the mass of the H-rich stellar envelope and $\tau_{\text {evol }}$ its evolutionary time scale; this relation has actually been verified for massive main sequence stars (Langer 1998; cf. Sect. 3). 


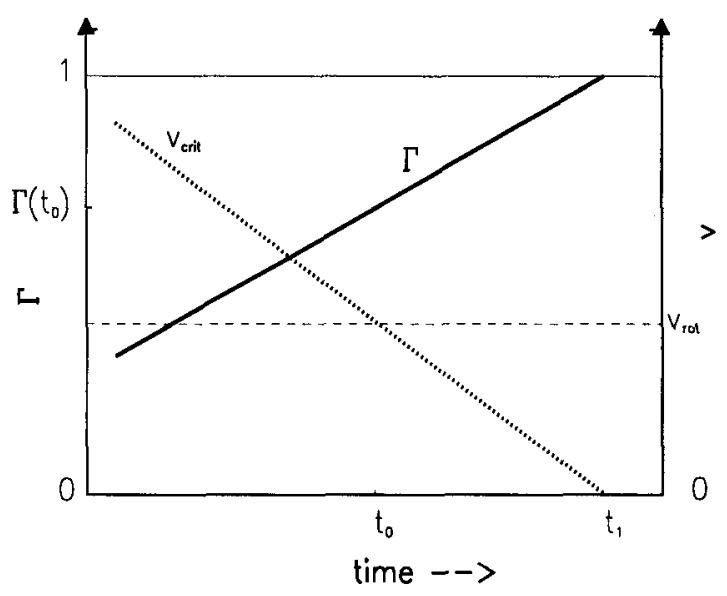

Fig. 1. Schematic time evolution of the Eddington factor $\Gamma=L / L_{\mathrm{Edd}}$ (thick line), with Eddington luminosity $L_{\mathrm{Edd}}=4 \pi c G M / \kappa$, where $c$ is the speed of light, $M$ and $L$ are mass and luminosity of the star, and $\kappa$ is the opacity at the stellar surface, and of the critical rotation velocity $v_{\text {crit }}=[(1-\Gamma) G M / R]^{1 / 2}$ (dotted line), where $R$ is the radius of the star. For simplicity, we assumed a constant equatorial rotational velocity $v_{\text {rot }}$ (dashed line); however, a time dependence of $v_{\text {rot }}$ would not alter the conclusions. The curves shown are for a massive star expanding at constant luminosity $L$, which - in the non-rotating case - would reach the Eddington limit due to an opacity increase in its surface layers at $t=t_{1}$. However, since the rotational velocity $v_{\text {rot }}$ must always be finite, the critical rotation, where the combination of radiation pressure and centrifugal force exceeds surface gravity at the equator, occurs at a time $t_{0}<t_{1}$, i.e. before the Eddington factor reaches the critical value of $\Gamma=1$. Thus, outflows occur before the star reaches its Eddington limit, acting to prevent further expansion.

\section{Core hydrogen burning}

Very luminous stars may approach their $\Omega$-limit during core hydrogen burning. Langer (1998) has shown that this is not connected with any catastrophic mass loss, but that instead the coupling between mass and angular momentum loss leads to a stable, long-lasting evolutionary stage at the $\Omega$-limit (cf. Fig. 2). During this stage, the mass loss is increased (by roughly a factor 10 for a $60 \mathrm{M}_{\odot}$ example) and stable, but strongly unisotropic. Langer \& Heger (1998) proposed that the luminous B[e] supergiants (Gummersbach et al. 1995) may correspond to this stage.

The question as to stars of which mass, metallicity and initial rotation rate do actually reach the $\Omega$-limit during core hydrogen burning is far from settled (cf. Langer 1998). It is intriguing that the so called Pistol Star close to the Galactic Center (Figer et al. 1998), which appears to have an initial mass well above $150 \mathrm{M}_{\odot}$, is best understood with the assumption that rotation did not affect its evolution. In fact, its location in the HR diagram, in particular 


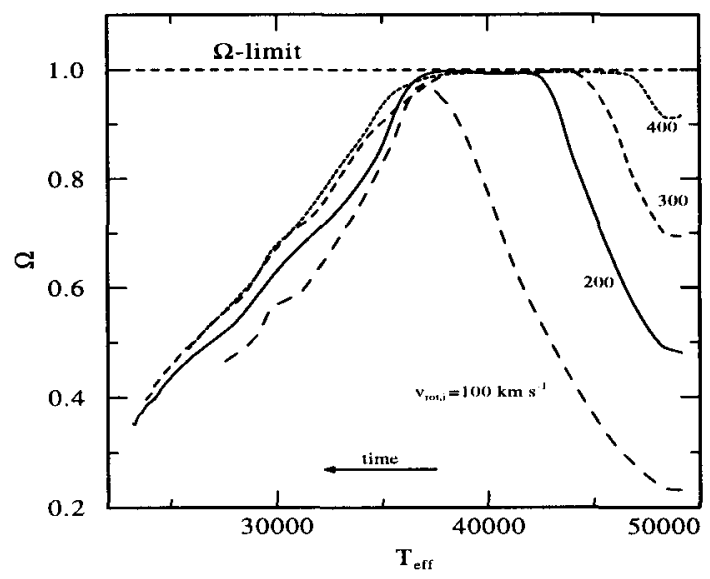

Fig. 2. $\Omega$ as function of the effective temperature during core hydrogen burning, for $60 \mathrm{M}_{\odot}$ sequences with different initial rotation rates (cf. Langer 1998).

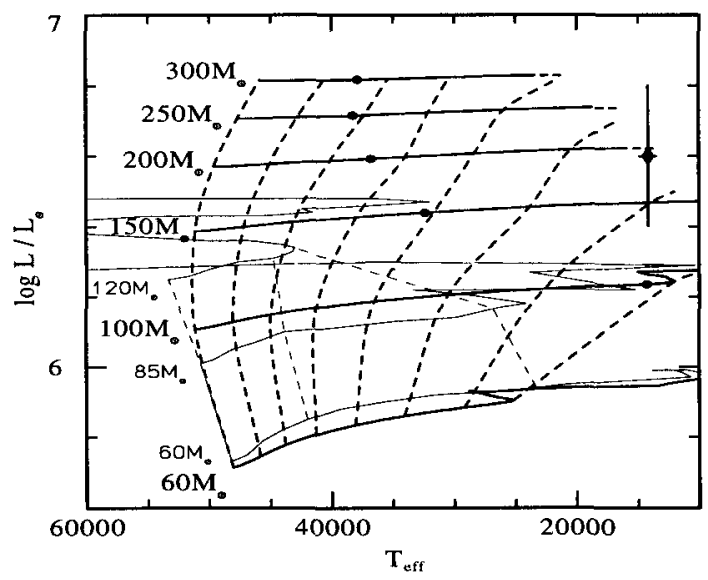

Fig. 3. Stellar evolutionary tracks in the HR diagram for core H-burning non-rotating stars in the initial mass range $60 \ldots 300 \mathrm{M}_{\odot}$ and a metallicity $\mathrm{Z}$ of $2 \%$ (thick continuous lines). Thick dashed lines connect models with central helium mass fractions of $0.28,0.4,0.5, \ldots, 0.9$, and 0.98 . Black dots mark the first appearance of hydrogen burning products at the stellar surface. The tracks of the 200,250 , and $300 \mathrm{M}_{\odot}$ models end due to the occurrence of surface instabilities. Thin continuous lines show evolutionary tracks for 60,85 , and $120 \mathrm{M}_{\odot}$ and $Z=0.02$ obtained by Schaller et al. (1992) - without their effective temperature correction - who included convective core overshooting, with thin dashed lines connecting models with central helium concentrations of $0.30,0.60$, and 0.90 . The position of the Pistol Star is marked by a diamond, together with the error bar (cf. Figer et al. 1998). 


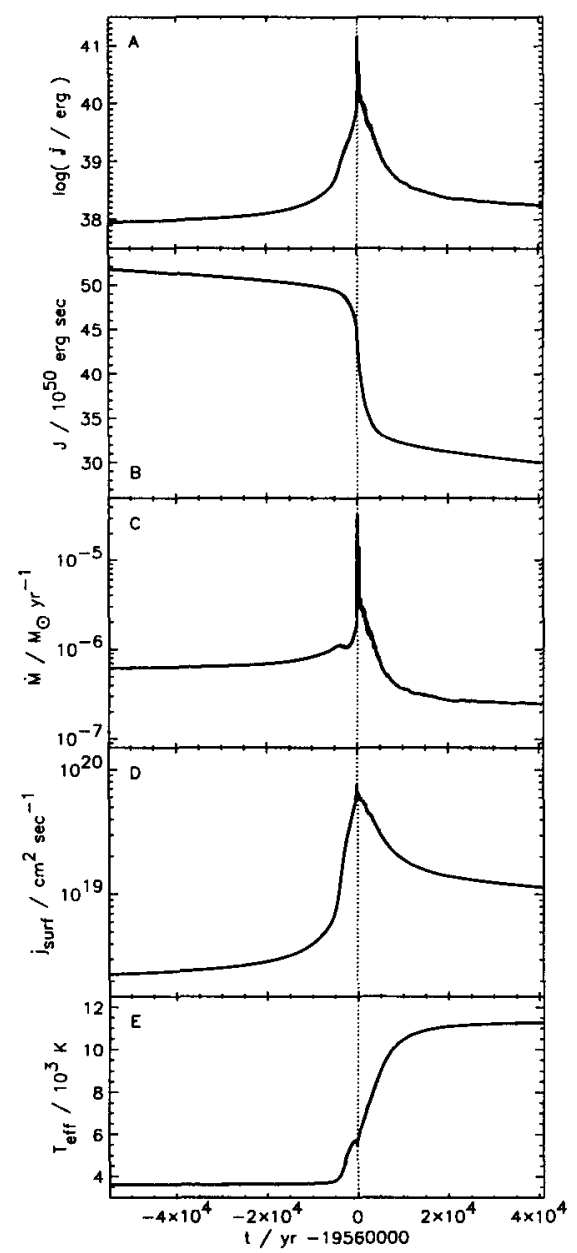

Fig. 4. Evolution of characteristic stellar properties as function of time, during the first part of the blue loop of a $12 \mathrm{M}_{\odot}$ model (cf. Heger \& Langer 1998). The time zero-point is arbitrarily defined. Displayed are: the angular momentum loss rate $\dot{J}(\mathbf{A})$, the total angular momentum $J(B)$, the mass loss rate $\dot{M}(\mathrm{C})$, the specific angular momentum loss rate $\dot{J} / \dot{J}=j_{\text {surf }}$ (D), and the effective temperature $\log T_{\text {eff }}(\mathbf{E})$.

its low effective temperature, seems to exclude any extra mixing as produced by rotation or even convective core overshooting (cf. Fig. 3).

\section{Luminous Blue Variables}

The scenario of the $\Omega$-limit can lead to an understanding of several essential features of the Luminous Blue Variables (LBVs). First of all, it is at the least conceivable that very massive stars arrive at their $\Omega$-limit directly after core hydrogen exhaustion, since the ensuing envelope expansion leads to stellar effective temperatures where the helium opacity peak appears close to the surface and thus the Eddington factor $\Gamma$ becomes close to one. According to Sect. 2, we can then expect the star to produce very high mass loss rates due to the small evolutionary time scale of the envelope, and the mass loss 


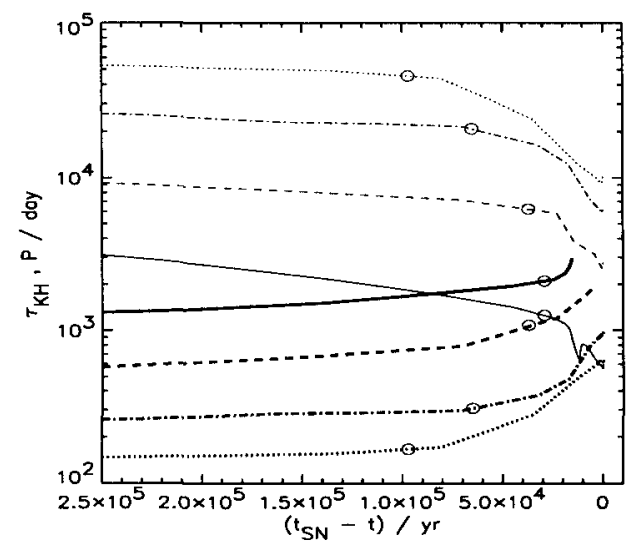

Fig. 5. Kelvin-Helmholtz time scale of the H-rich stellar envelope (thin lines) as function of the time left until the supernova explosion of the star, for the computed 10 (dot), 12 (dash-dot), 15 (dash), and $20 \mathrm{M}_{\odot}$ (solid line) sequences. The thick lines show the period of the fundamental mode as derived from a linear stability analysis. Small circles designate the time when the central helium mass fraction has reached $1 \%$, i.e. roughly central helium exhaustion. It is evident that, at least for the 15 and $20 \mathrm{M}_{\odot}$ sequences, pulsation periods of the order of the Kelvin-Helmholtz time scale occur. Note that for the last models of these two sequences linear pulsation periods could not be derived due to strong departures from thermal and hydrostatic equilibrium.

may be better designated eruption than wind (see also Langer 1997). Due to rotation, i.e. at the $\Omega$-limit, the circumstellar nebulae formed in this phase are expected to be strongly bipolar (Langer et al. 1998), which appears to be realized in practically all observed cases (Nota et al. 1995), $\eta$ Carinae being a well known example. Finally, the dependence of the $\Omega$-limit on the stellar rotation rate offers an explanation for the low luminosity of some LBVs: those would be the initially rapid rotators (Langer 1997, 1998).

\section{Core helium burning and beyond}

During core helium burning, massive stars would be red supergiants, blue supergiants or Wolf-Rayet stars. While most (though not all) Wolf-Rayet stars are expected to have spun down due to excessive mass and angular momentum loss (Fliegner \& Langer 1994), highly anisotropic mass loss can be expected for blue and even for some red supergiants. Heger \& Langer (1998) have found that the surface specific angular momentum (not only the surface rotation rate) can increase by more than one order of magnitude during the transition from the Hayashi line to hotter surface temperatures in the HR diagram (cf. Fig. 4). This can lead to circumstellar rings or bipolar structures 
around blue supergiants, perhaps such as found around the SN 1987A progenitor or B[e] supergiants (cf. Langer \& Heger 1998), and may already lead to anisotropic mass loss on the RSG branch, for which VY CMa may be a candidate (Wittkowski et al. 1999).

Finally, note that even though the statistical probability to observe this is small, massive red supergiants may produce highly variable and even unstable mass outflows shortly before they explode as Type II supernova (cf. Fig. 5), as their pulsation periods become of the same order as the thermal time scale of their envelopes (Heger et al. 1997).

Acknowledgements The author is very grateful to Peter Conti, Guillermo García-Segura, Alexander Heger, André Maeder, and Stan Owocki for enlightening discussions. This work has been supported by the Deutsche Forschungsgemeinschaft through grant No. La 587/15-1 and La 587/16-1.

\section{References}

Cassinelli J.P., Lamers H.J.G.L.M., 1998, Introduction to Stellar Winds, Cambridge University Press

García-Segura G., Mac Low M.-M., Langer N. 1996a, A\&A 305, 229

García-Segura G., Langer N., Mac Low M.-M. 1996b, A\&A 316, 133

Glatzel W., 1998, preprint

Gummersbach C.A., Zickgraf F.-J., Wolf B. 1995, A\&A, 302, 409

Figer D.F., Najarro F., Morris M., McLean I.S., Geballe T.R., Ghez A.M., Langer N.: 1998, ApJ, in press

Fliegner J., Langer N., 1994, in: Wolf-Rayet Stars: Binaries, Colliding Winds, Evolution, Proc. IAU-Symp. No. 163, K.A. van der Hucht et al., ed., p. 326

Friend D.B., Abbott D.C., 1986, ApJ 311, 701

Heger A., Jeannin, L., Langer, N., Baraffe, I. 1997, A\&A, 327, 224

Heger A., Langer N. 1998, A\&A 334, 210

Kippenhahn R. 1977, A\&A 58, 267

Langer, N. 1997, in: Luminous Blue Variables: Massive Stars in Transition, ed. A. Nota, H.J.G.L.M. Lamers., ASP Conf. Series, 120, 83

Langer, N. 1998, A\&A, 329, 551

Langer, N., Heger A., 1998, in B[e] stars, A.M. Hubert and C. Jaschek, eds., Kluwer, p. 235

Langer N., García-Segura G., Mac Low M.-M. 1998, ApJ, submitted

Nieuwenhuijzen H., de Jager C., 1995, A\&A 302, 811

Nota A., Livio M., Clampin M., Schulte-Ladbeck R., 1995, ApJ 448, 788

Owocki S.P., Cranmer S.R., Gayley K.G. 1996, ApJ 472, L151

Owocki S.P., Gayley K.G. 1998, proc. $2^{\text {nd }}$ Boulder-Munich Workshop on Hot Stars, ed. I. Howarth, A.S.P. Conf. Ser. Vol. 131, p. 237

Schaller G., Schaerer D., Meynet G., Maeder A., 1992, A\&AS 96, 269

Wittkowski M., Langer N., Weigelt G., A\&A, submitted

von Zeipel, H. 1924, MNRAS, 84, 665 


\section{Discussion}

R. Kudritzki: Your scenario of evolution at critical rotation in LBV-phases or the blue-supergiant phase could be checked by looking at observed rotational velocities obtained from echelle spectra. Have you already looked at this?

N. Langer: I have tried, but with ambiguous results. The problem is complicated by the fact that a critical rotator does not have to be a rapid rotator since, e.g., close to the Eddington limit the critical rotational velocity goes to zero. However, more observational determinations of $v_{\text {rot }}$ could shed light on this question in a statistical sense.

F.-J. Zickgraf: You showed that the first time a star reaches the $\omega$-limit, it might stay there for up to $10^{5}$ years. Interestingly, this agrees with an estimate for the duration of the $\mathrm{B}[\mathrm{e}]$ phase based on the ratio of main sequence stars to $\mathrm{B}[\mathrm{e}]$ stars. Is the time scale at the second time of reaching the $\omega$-limit significantly shorter?

N. Langer: Yes it is, since the post-main sequence evolutionary time scale is much shorter than that on the main sequence.

G. Koenigsberger: In the case of a star in a binary system, one gets even larger possible mass loss rates. Could these rates be so large that the star is not able to go to cooler effective temperatures and remains at an early spectral type even during the LBV phase?

N. Langer: Yes. For a given luminosity, the minimum effective temperature can be estimated through the "radius" of the Roche lobe via the StefanBoltzmann law. Even though the mass-loss mechanism is different (i.e., Roche lobe overflow) from the mechanism for single stars, one may still expect an LBV-type outburst behaviour if the primary star (the mass loser) is in the immediate post-core H-burning phase.

S. Owocki: A general comment: I suggest we think of gravity darkening not just in terms of a simple parameterisation, but in terms of the fundamental physical effect: namely, diffusing photons will always tend to escape in the direction of least resistance, which for an oblate star is from the pole. Even if one were to avoid gravity darkening, it would be hard to use radiation to support an equatorial "ring" of higher density, simply because the radiative flux will avoid such high density.

R. Humphreys: Regarding the origin of the bipolar rings around SN 1987A: an evolved, mass-losing $\mathrm{M}$ supergiant - VY $\mathrm{CMa}$ - is bipolar and probably has an equatorial disk of dust.

N. Langer: Yes. In a recent paper with M. Wittkowski and G. Weigelt (see references), we argue that VY CMa may in fact be at the stage of leaving the Hayashi line, i.e., an immediate progenitor of IRC +10420 - which has 
bipolar outflows - and already shows the spin-up effect recently found by Heger \& Langer (1998).

R. Schulte-Ladbeck: Could you comment on the implications of your results for the B/R supergiant ratio of galaxies? I have an approved HST programm to revisit this problem.

N. Langer: In a recent paper, A. Maeder and I $(1995$, A\&A 195, 685) have shown that present stellar evolution models cannot predict the $B / R$ ratio as a function of metallicity correctly. Whether this changes when the effects of rotation are included is not yet known. However, it would be nice to have improved observational data to investigate this question.

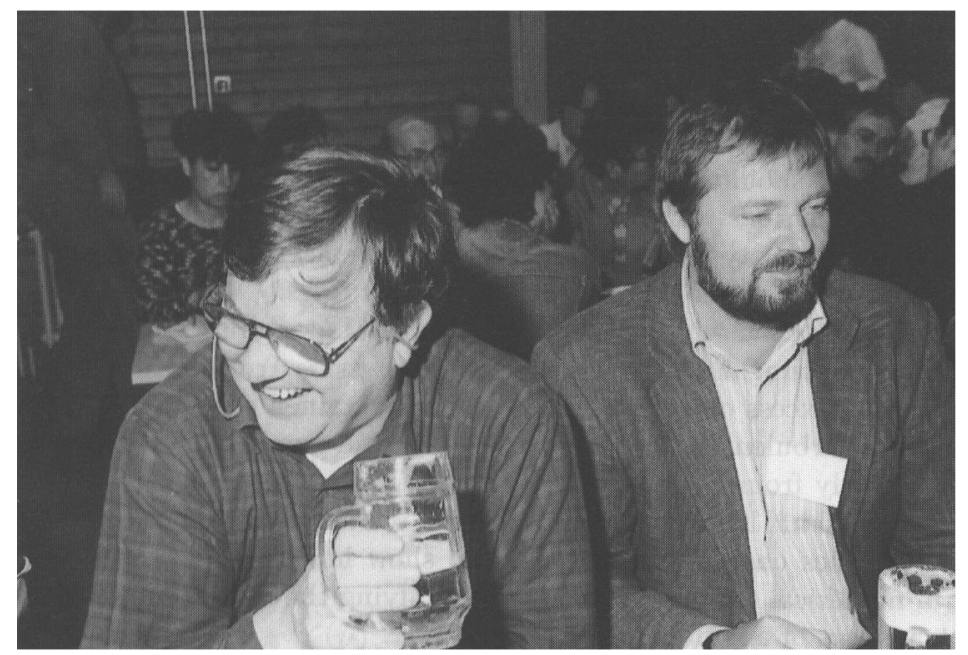

Peredur Williams and Norbert Langer 